\title{
Acessibilidade e mobilidade urbana: $O$ ambiente construído como instrumento do direito fundamental de ir e vir
}

Accessibility and urban mobility: The environment built as an instrument of the fundamental right

to come and go

Accesibilidad y movilidad urbana: el medio ambiente construido como instrumento del derecho

fundamental de ir y venir

Emanuel Rodrigo Reis da Silva ORCID: https://orcid.org/0000-0002-1626-0611

Universidade Federal do Maranhão, Brasil E-mail: rodrigoreis405@gmail.com

Humberto Denys de Almeida Silva ORCID: https://orcid.org/0000-0002-3144-7070 Universidade Federal do Piauí, Brasil E-mail: hdas0912@hotmail.com

Samuel Jônatas de Castro Lopes ORCID: https://orcid.org/0000-0002-9520-414X Universidade Federal do Piaú, Brasil E-mail: samueljonatas09@gmail.com

Hitalo de Jesus Bezerra da Silva ORCID: https://orcid.org/0000-0001-6008-3600 Universidade Federal do Piaú, Brasil E-mail: hitalo.ufpi@gmail.com

Raiany Sena de Oliveira

ORCID: https://orcid.org/0000-0003-4623-8156 Centro Universitário Maurício de Nassau, Brasil

E-mail: raianysena-oliveira@hotmail.com

Antônio Alves de Carvalho Júnior ORCID: https://orcid.org/0000-0002-7552-6475 Instituto Federal do Piauí, Brasil E-mail: carvalho.junior@ifpi.edu.br

Kelson de Sousa Leite

ORCID: https://orcid.org/0000-0003-2772-452X Instituto Federal do Piauí, Brasil E-mail: kelson.leite@ifpi.edu.br Linardy de Moura Sousa ORCID: https://orcid.org/0000-0002-3396-8080

Centro Universitário Santo Agostinho, Brasil E-mail: linardy_moura@hotmail.com

Valdivânia Albuquerque do Nascimento ORCID: https://orcid.org/0000-0002-3188-4676 Universidade Federal do Piauí, Brasil E-mail: val.albuquerque@hotmail.com

Laecio Guedes do Nascimento ORCID: https://orcid.org/0000-0003-3321-8156 Centro Universitário Santo Agostinho, Brasil E-mail: laecioguedes25@hotmail.com

Amanda Fernandes Pereira da Silva ORCID: https://orcid.org/0000-0001-7616-8166 Universidade Federal do Piauí, Brasil

E-mail: amandafeernandes09@gmail.com

Denise Rodrigues Santiago

ORCID: https://orcid.org/0000-0003-1952-7913 Universidade: Federal do Piauí, Brasil E-mail: arq.denise15@gmail.com

Samuel Campelo Dias ORCID: https://orcid.org/0000-0002-4357-515X Universidade Federal do Piauí, Brasil E-mail: samuel.c.dias@hotmail.com 


\author{
Jessica Waleska Dias Pires \\ ORCID: https://orcid.org/0000-0001-9389-2404 \\ Universidade Brasil, Brasil \\ E-mail: jessica-waleska@hotmail.com \\ Paulo Henrique Nogueira da Silva Filho \\ ORCID: https://orcid.org/0000-0002-6146-5616 \\ Centro de Ensino Superior Vale do Parnaíba, Brasil \\ E-mail: nogueiraengenharia1@gmail.com
}

\begin{abstract}
Resumo
É dever do Estado promover o bem comum sem quaisquer formas de discriminação, a fim de que seja cumprida com eficiência a função social da cidade, tendo em vista que tal função constitui-se pela seguridade de que todos gozem de moradia, do lazer, dos serviços públicos e da circulação nas vias. Entretanto, a presença de barreiras impostas por projetos arquitetônicos equivocados ainda é bastante comum nas edificações do país. Assim, assegurar a cidadania e a dignidade de todos os brasileiros resulta em vislumbrar no projeto arquitetônico as edificações como instrumentos garantidores do direito de ir e vir. A partir desta perspectiva, essa pesquisa teve por objetivo discutir a acessibilidade como instrumento de inclusão social no ambiente construído, de modo que se evidencie o préstimo de se considerar a acessibilidade universal como direito fundamental. Adotou-se como metodologia de investigação o modelo de pesquisa bibliográfica de natureza qualitativa. Desta forma, foi possível concluir que a garantia de um ambiente acessível é de grande importância para a vida social das pessoas portadoras de necessidades especiais ou com mobilidade reduzida, pois promove qualidade de vida e garante o exercício pleno de sua cidadania.
\end{abstract}

Palavras-chave: Acessibilidade; Mobilidade urbana; Arquitetura acessível; Direito de ir e vir.

\begin{abstract}
It is the State's duty to promote the common good without any form of discrimination, so that the social function of the city is efficiently fulfilled, considering that such function is constituted by the security that everyone enjoys housing, leisure, public services and traffic on the roads. However, the presence of barriers imposed by mistaken architectural projects is still quite common in the country's buildings. Thus, ensuring the citizenship and dignity of all Brazilians results in seeing buildings in the architectural project as instruments that guarantee the right to come and go. From this perspective, this research aimed to discuss accessibility as a tool for social inclusion in the built environment, in order to highlight the usefulness of considering universal accessibility as a fundamental right. The research methodology adopted was the qualitative bibliographic research model. Thus, it was possible to conclude that the guarantee of an accessible environment is of great importance for the social life of people with special needs or with reduced mobility, as it promotes quality of life and guarantees the full exercise of their citizenship.
\end{abstract}

Keywords: Accessibility; Urban mobility; Accessible architecture; Right to come and go.

\title{
Resumen
}

Es deber del Estado promover el bien común sin ningún tipo de discriminación, para que la función social de la ciudad se cumpla de manera eficiente, considerando que dicha función está constituida por la seguridad de que todos disfruten de la vivienda, el esparcimiento, los servicios públicos y el tráfico en la ciudad. carreteras. Sin embargo, la presencia de barreras impuestas por proyectos arquitectónicos equivocados sigue siendo bastante común en los edificios del país. Así, asegurar la ciudadanía y la dignidad de todos los brasileños resulta en ver los edificios en el proyecto arquitectónico como instrumentos que garantizan el derecho de ir y venir. Desde esta perspectiva, esta investigación tuvo como objetivo discutir la accesibilidad como herramienta de inclusión social en el entorno construido, con el fin de resaltar la utilidad de considerar la accesibilidad universal como un derecho fundamental. La metodología de investigación adoptada fue el modelo de investigación bibliográfica cualitativa. Así, se pudo concluir que la garantía de un entorno accesible es de gran importancia para la vida social de las personas con necesidades especiales o con movilidad reducida, ya que promueve la calidad de vida y garantiza el pleno ejercicio de su ciudadanía.

Palabras clave: Accesibilidad; Movilidad urbana; Arquitectura accesible; Derecho de ir y venir.

\section{Introdução}

A Constituição Federal da República do Brasil, promulgada em 05 de outubro de 1988, no seu artigo primeiro, ressalta dentre os fundamentos constitucionais da república: a cidadania e a dignidade da pessoa humana, assegura ainda, no artigo terceiro, que é dever do Estado promover o bem comum sem quaisquer formas de discriminação. O texto versa no artigo quinto acerca da inviolabilidade, dentre outros, do direito à liberdade, à igualdade e à segurança (Brasil, 1988).

O Decreto Federal n. ${ }^{\circ}$ 5.296, promulgado em 08 de dezembro de 2004, que regulamenta as Leis Federais n. ${ }^{\circ} 10.048$, expedida em 08 de novembro de 2000 (Brasil, 2000a), e n. ${ }^{\circ}$ 10.098, publicada em 19 de dezembro de 2000 (Brasil, 2000b), 
determina em seus artigos 10 e 11 que, para aprovação, licenciamento ou emissão de certificados de conclusão de projetos arquitetônicos ou urbanísticos, construção, ampliação, ou mudanças de destinação de edificações, deverá ser observada a disponibilidade de acessibilidade das mesmas, de modo que essas tornem-se ou sejam acessíveis (Brasil, 2004a).

Elaborada em 1985 pelo Comitê Brasileiro 40 da Associação Brasileira de Normas Técnicas - ABNT, a NBR 9050 teve sua última revisão em 2020 (ABNT 9050, 2020), de modo a garantir um arcabouço consultivo capaz de orientar a criação de condições para a concepção arquitetônica de ambientes favoráveis à circulação de quaisquer indivíduos (Fávero, 2004), ou seja, com o condão do conceito de desenho universal.

De acordo com Santos (1988):

O espaço deve ser considerado como um conjunto indissociável de que participam de um lado certo arranjo de objetos geográficos, objetos naturais e objetos sociais, e, de outro, a vida que preenche e os anima, ou seja, a sociedade em movimento (Santos, 1988, p. 26-27).

Neste contexto, as informações transmitidas pela normatização de locomobilidade aos projetistas vão deste da métrica dos elementos construtivos até os parâmetros de adequação e disposição das sinalizações, acessos, circulação e arranjo dos mobiliários urbanos (Silva, 2019). Apregoa, neste dispositivo, que as edificações e os espaços urbanos sejam compostos de rotas acessíveis e livres de objeções. A Figura 1, segundo a ABNT 9050 (2020), expõe um trajeto ininterrupto, livre de barreiras e com sinalização adequada, que tem como finalidade conectar os ambientes internos e externos dos empreendimentos, bem como garantir a segurança e autonomia do usuário.

Figura 1. Rota acessivel.

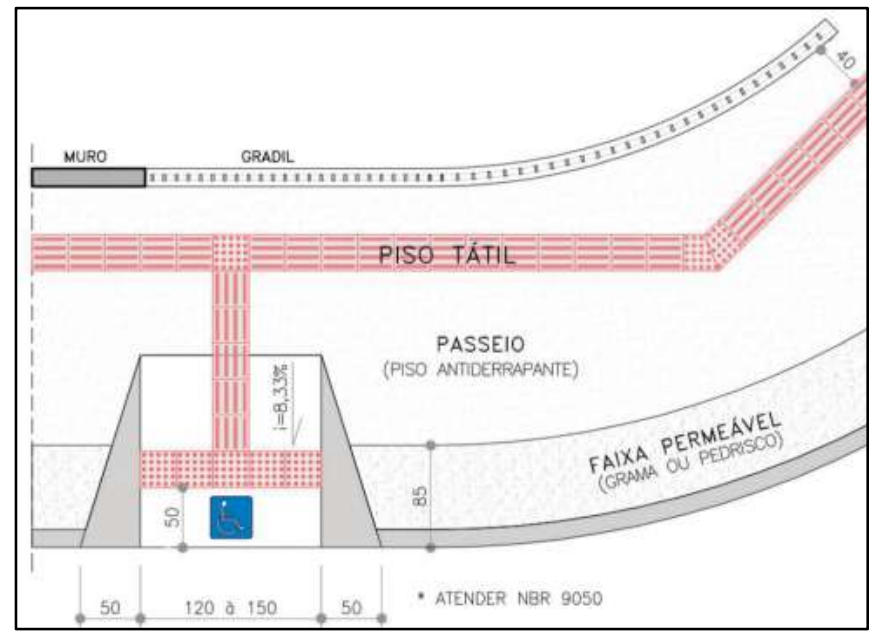

Fonte: Cartilha de acessibilidade, CREA SP (2019).

Quanto às condições gerais para mobiliários urbanos, estes devem atender aos princípios do desenho universal, ou seja, proporcionar ao usuário seguridade e independência, de modo que tenham dimensões e espaços apropriados para aproximação, alcance, manipulação e uso, devendo ser projetados de maneira que não sejam, posteriormente, constituídos como obstáculos suspensos (ABNT 9050, 2020), como visto na Figura 2. 
Figura 2. Modelo de instalação de mobiliario urbano.

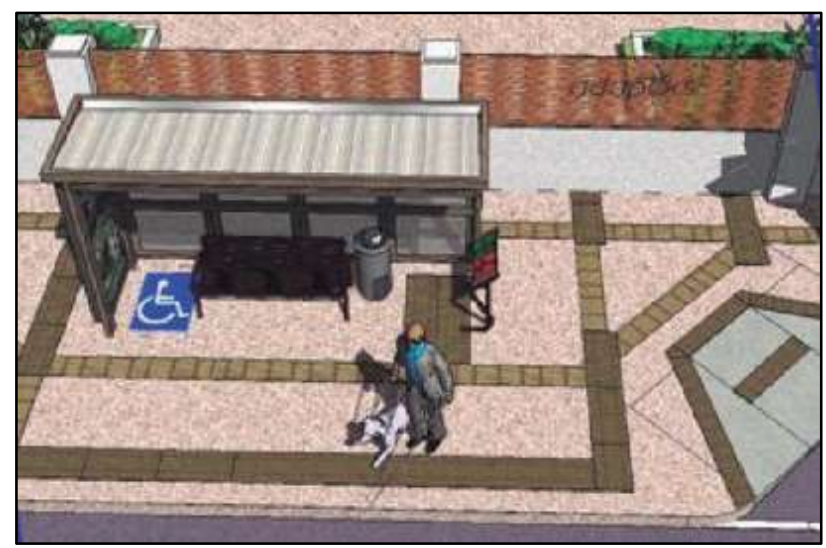

Fonte: Cartilha de acessibilidade, CREA SP (2019).

Neste contexto, respaldados pela normatização consultiva de cunho técnico, bem como pelo o arcabouço jurídico brasileiro, Duarte e Cohen (2004) refletem sobre a exclusão social que se concretiza quando os espaços se tornam práticas sociais marginalizatórias, sem o menor valor às diferenças, estando estes como agentes de um apartheid. Assim, para o Ministério das Cidades (Brasil, 2004b), é vital pensar insistentemente a urbe como lugar para todos, como atesta Lanchotti (2016), todas as políticas públicas estão obrigadas a uma tentativa de minimização ou neutralização dos efeitos negativos dos impedimentos ou limitações impostas aos indivíduos no que no que se refere à falta de acessibilidade na mobilidade urbana.

Desta maneira, é notória a relevância da ABNT 9050 (2020) para o cenário da acessibilidade no Brasil, haja vista que há através deste dispositivo uma busca pela inclusão de uma parcela significativa da população do país na vivência social das cidades, a julga por, que os critérios e parâmetros técnicos preconizados neste normativo visam proporcionar diferentes condições de locomobilidade e percepção dos ambientes (Calado, 2006), o que para Santos Filho (2010), torna a norma brasileira de acessibilidade eficaz e eficiente no que cerne a eliminação de barreiras nos espaços construídos.

Neste sentido, Quaresma e Romita (2001) apontam que a única forma de promover o equilíbrio coletivo é por meio da inclusão, ou seja, permitir um tratamento igualitário aos desiguais na medida exata de suas desigualdades (Botelho, 2008) o que, como reflete Araújo (2007), nada mais é que propiciar aos que necessitam as ferramentas adequadas para gozarem das mesmas condições dos demais indivíduos.

Sestaro (2008) afirma que um ambiente inacessível pode ser considerado como um mecanismo de discriminação, haja vista que a ausência de adequação dos espaços restringe, desencoraja e impede a participação e a inserção da pessoa com deficiência na vida comunitária do burgo, ficando este ser impedido do completo exercício do direito de ir e vir (Niess, 2003), e dos demais direitos fundamentais inerentes ao cidadão: saúde, educação, lazer, trabalho, segurança, assistência, proteção e outros (Leite, 2007).

Diante do exposto, o presente trabalho discutirá acessibilidade como instrumento de inclusão social no ambiente construído, apresentado os principais parâmetros da normatização técnica de mobilidade vigente no Brasil, bem como evidenciando o préstimo para o coletivo social de se considerar a acessibilidade universal como direito fundamental na produção arquitetônica de logradouros, passeios e edifícios públicos e privados das cidades brasileiras.

\section{Metodologia}

Nesta seção desenvolve-se uma narrativa da metodologia que foi utilizada nesse trabalho, descrevendo os procedimentos utilizados, a fim de se chegar na obtenção dos resultados. De acordo com Vergara (2005), as pesquisas classificam-se levando em consideração duas percepções fundamentais ao estudo de cunho cientifico, que são: a ótica dos fins e a dos meios. 
Neste contexto, Gil (2008) declara que existem diferentes modelos de pesquisa. Desta forma, a presente obra configurase como um estudo teórico no qual foi adotado como tipologia de exame o modo aplicado de pesquisa de método indutivo. Quanto aos objetivos, tal pesquisa classifica-se como explicativa de natureza qualitativa, haja vista que este formato se caracteriza pela identificação e qualificação como meio de examinar indutivamente o conteúdo abordado (Lakatos \& Marconi, 1996), o que no trabalho em questão dar-se-á pelo anseio de compreender a alcançabilidade como direito inegável, indelével e garantidor de um tratamento isonômico ao cidadão brasileiro. Já em relação à metodologia discricional, optou-se por realizar um estudo bibliográfico.

De acordo com Vergara (2005), a pesquisa bibliográfica constitui-se pela averiguação de materiais já elaborados acerca de uma temática, à vista disso, a pesquisa ora desenvolvida dividiu-se em duas etapas: a primeira consistiu no levantamento bibliográfico, o que nesse trabalho tomou-se como fonte de dados consultivos o normativo técnico da ABNT que versa sobre acessibilidade, bem como as fontes literárias e jurídicas disponíveis sobre o tema, dando total enfoque na acessibilidade arquitetônica nos espaços com o objetivo de conhecer e analisar as contribuições científicas do passado existente sobre o assunto. Foram utilizados no estudo: artigos, leis e norma datados entre 1988 e 2020.

Foram considerados como principais autores para o levantamento de dados deste trabalho: Almeida (2018), Araújo (2007), Botelho (2008), Carvalho e Castro (2012), Condorcet (2006), Duarte e Cohen (2004), Fávero (2004), Feijó (2002), Gomide e Galindo (2013), Lanchoti (2016), Leite (2007), Leite (2013), Miotti (2012), Niess e Niess (2003), Quaresma e Romita (2001), Santos (1988), Sestaro (2008) e Silva (2019).

Já na segunda etapa, iniciou-se a análise dos dados oriundos das informações sobre a acessibilidade e a mobilidade urbana no cenário brasileiro por meio de uma verificação exploratória dos artigos e leis que foram pré-selecionados para a pesquisa, o que de acordo com Gil (2008), proporciona ao pesquisador uma visão ampla do estágio em que se encontram as informações já disponíveis sobre o assunto, bem como maior familiaridade com o problema, de maneira que se torna possível alcançar as respostas à temática deste artigo.

\section{Resultados e Discussão}

O Engenheiro Civil Carvalho e Castro (2012), apregoa que os mecanismos que norteiam as condições ideais de acessibilidade não são indecifráveis, inacessíveis e tão pouco inatingíveis, alegar ignorância acerca desses instrumentos, como atenuante para o desrespeito ao direito de acessibilidade, torna-se declaração incoerente e descabida. Entretanto, a presença de barreiras impostas por projetos arquitetônicos equivocados ainda é bastante comum nas edificações e logradouros do país (Almeida, 2018) tendo em vista que são vertentes facilitadoras desses equívocos: a pouca instrução da população a respeito do assunto, e a precária fiscalização realizada pelos órgãos públicos.

Nesta perspectiva, Miotti (2012) versa que entre as formas de irreverência ao direito de ir e vir nas cidades brasileiras, são as mais encontradas: rampas deterioradas, piso irregulares e inadequados, degraus, árvores com raízes expostas, e calçadas e passeios em má conservação, como mostrado na Figura 3. 
Figura 3. Principais barreiras à acessibilidade encontradas nas cidades brasileiras.

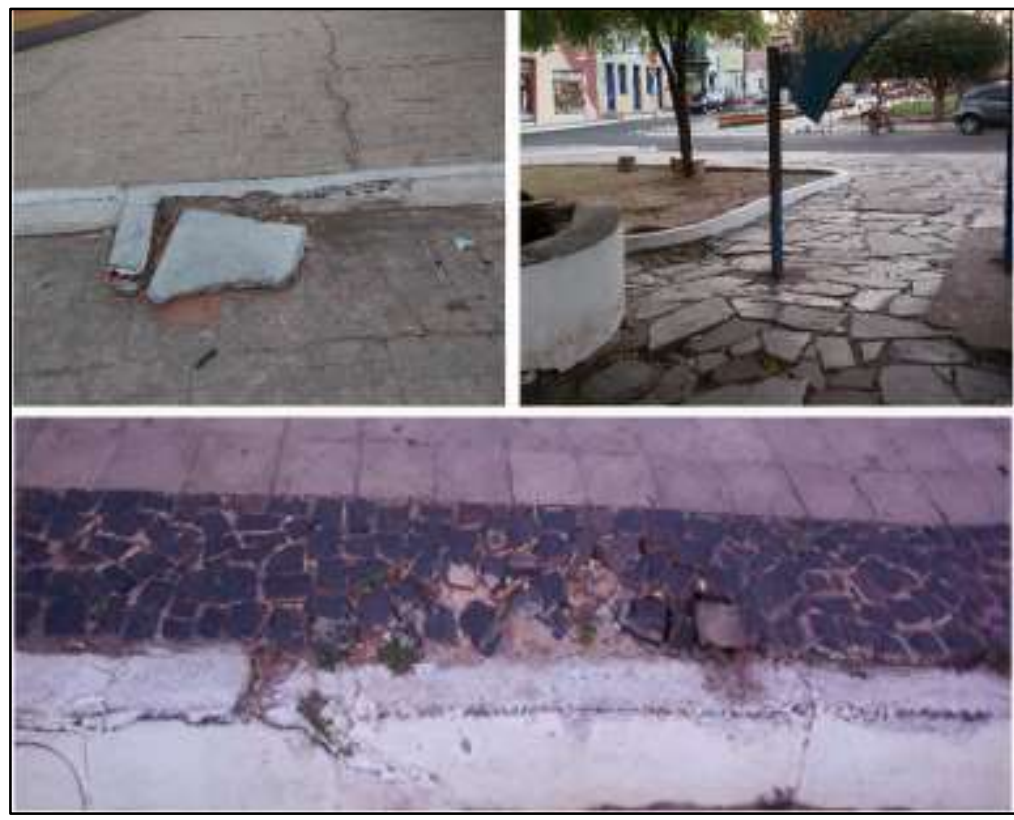

Fonte: Almeida (2018).

Ao refletir sobre a qualidade da transitabilidade oferecida nos ambientes e logradouros do país, verificou-se que apesar da existência de um elevado volume de fontes bibliográficas técnicas que tratam acerca das prerrogativas essenciais inerentes a este campo, há ainda nas cidades e edificações brasileiras uma vasta necessidade de adequações para que estas se tornem totalmente acessíveis, a julgar por, que o Instituto Brasileiro de Geografia e Estatística (IBGE, 2010), quantificou que cerca de 42,6\% dos prédios públicos que comportam as prefeituras nas cidades brasileiras não contam com dispositivos de acessibilidade.

Deste modo, a inexistência de acessibilidade agrava os indicadores de segregação social à medida que impossibilita o acesso de determinado seguimento populacional aos serviços públicos essenciais, ou seja, torna os espaços públicos instrumento propício a supressão dos cidadãos com mobilidade reduzida (Leite, 2013).

O que para Feijó (2002) dá-se pela não compreensão social do indivíduo portador de deficiência como sujeito culturalmente completo, de maneira que se torna evidente que os impedimentos condicionados a mobilidade não estão na pessoa com necessidades especiais, porém, na relação desta com a indisponibilidade de equiparação social através de um espaço urbano isonômico (Prado, 2010). Como atestado por Gomide e Galindo (2013), a locomobilidade urbana sustentável é definida como aquela capaz de promover o acesso isonômico das pessoas à cidade, seus serviços e oportunidades.

Sob essa ótica, abordar, discutir e evidenciar a importância da temática da acessibilidade para o campo da concepção arquitetônica dos ambientes permite a criação de mecanismos que evoquem o tratamento à acessibilidade como função pública, a fim de que novos espaços urbanos sejam projetados com qualidades que supram a necessidade dos indivíduos, como visto na Figura 4, bem como que as modificações inerentes a este campo sejam executadas nos espaços já existentes (Almeida, 2018). 
Figura 4. Exemplo de ambientes com acessibilidade adequada.

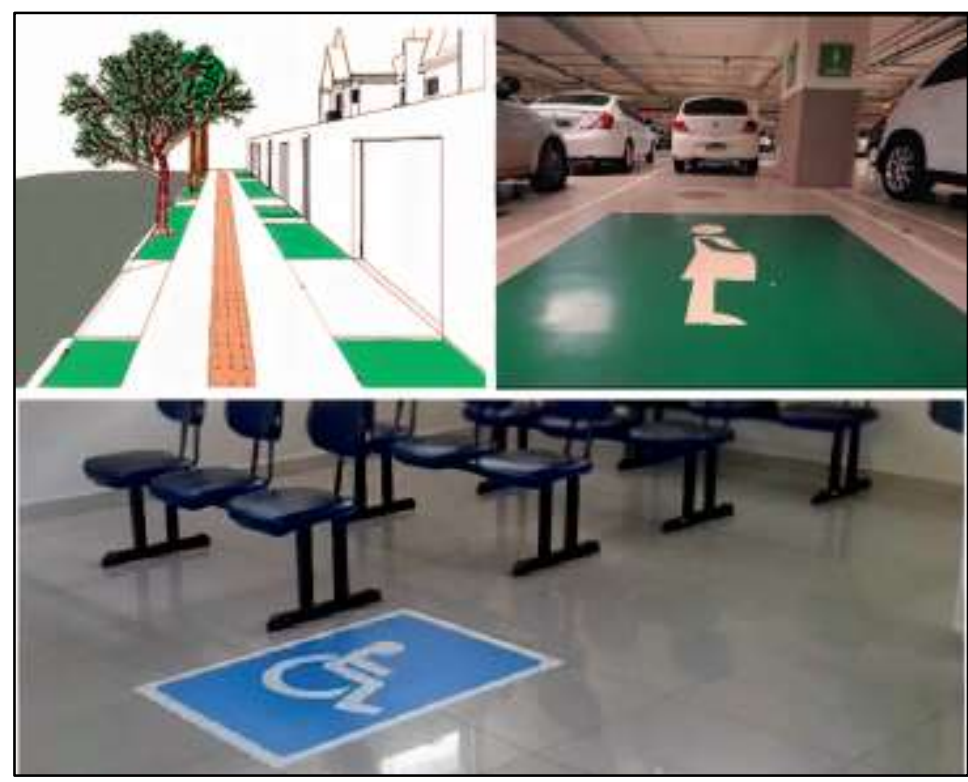

Fonte: Cartilha de acessibilidade, CREA SP (2019).

Destarte, torna-se coerente afirmar que os dispositivos de acessibilidade devem ser considerados como elementos construtivos obrigatórios na elaboração do projeto arquitetônico das edificações, a julgar por, que a aplicação das considerações de locomobilidade nas fases iniciais de projeto proporciona a diminuição da ociosidade dos espaços, bem como propicia a inclusão e a alcançabilidade total dos usuários desses locais, o que torna, de acordo com Condorcet (2006), a transitabilidade um instrumento de equiparação social hábil a reduzir os indicadores de desigualdade, minimizar as formas de preconceito e prover o bem comum.

À vista disto, tira-se como consequência que todos e quaisquer empreendimentos, públicos ou privados, estão obrigados a possibilitar condições de alcance, percepção e entendimento para utilização com segurança e autonomia de suas dependências, espaços e mobiliários, o que ocorre através da estrita observância da diretriz normativa peculiar ao tema em voga para projetos de engenharia e arquitetura, a norma técnica NBR 9050, de 03, de agosto, de 2020.

\section{Considerações Finais}

Seguindo o objetivo pré-estabelecido de levantar referências acerca da acessibilidade como instrumento de inclusão social no ambiente construído, notou-se que os autores são uníssonos em afirmar que os obstáculos que se apresentam adversos ao deslocamento acessível nos espaços não estão intrinsicamente relacionadas as deficiências ou limitações de seus usuários, entretanto, as barreiras encontradas nesses locais. Desta maneira, se torna visível e evidente que o ambiente construído deve atender as diversas indigências sociais, ou seja, deve ser projetado e executado com vistas ao desenho universal, a fim de acomodar as pessoas com eficiência, inclusive as com deficiência e mobilidade reduzida, em suas atividades corriqueiras.

Sendo assim, garantir um ambiente acessível é de grande importância às pessoas portadoras de necessidades especiais ou com mobilidade reduzida, pois promove qualidade de vida e garante o exercício pleno de cidadania. No entanto, há uma evidente falta de fiscalização por parte do poder público quanto ao estrito cumprimento das normas de transitabilidade nas produções arquitetônicas, de modo que não há o fornecimento de uma estrutura adequada e eficiente à acessibilidade no cenário urbanístico brasileiro, ficando o cidadão portador de necessidade especial ou mobilidade reduzida com o seu direito a locomoção isonômica tolhido. 


\section{Referências}

Almeida, A. S. C. (2018). Acessibilidade no espaço público: estudo de caso nas praças do centro da cidade de Coelho Neto - MA. 21 f. (Trabalho de Conclusão de Curso), Centro Universitário de Ciências e Tecnologia do Maranhão - UNIFACEMA, Caxias.

Araújo, L. A. D. (2007). A proteção constitucional das pessoas portadoras de deficiência. CORDE.

Associação Brasileira de Normas Técnicas - ABNT. (2020). NBR 9050: Acessibilidade a edificações, mobiliário, espaços e equipamentos urbanos. ABNT.

Botelho, J. (2008). A atuação do Ministério Público. Como interpretar o decreto federal e demais regulamentações da acessibilidade. Anais do Seminário “Os Desafios de Adequação à Lei de Acessibilidade”. MBA Treinamentos.

Brasil. (2019). Conselho Regional de Engenharia e Agronomia do Estado de São Paulo - CREASP. Cartilha de Acessibilidade. CREASP. http://www.creasp.org.br.

Brasil. (1988). Constituição da República Federativa do Brasil de1988. Saraiva.

Brasil. (2004). Decreto $n^{\circ}$. 5.296, de 08 de dezembro de 2004. Regulamenta as Leis no 10.048, de 8 de novembro de 2000 , que dá prioridade de atendimento às pessoas que especifica, e 10.098, de 19 de dezembro de 2000, que estabelece normas gerais e critérios básicos para a promoção da acessibilidade das pessoas portadoras de deficiência ou com mobilidade reduzida, e dá outras providências. http://www.planalto.gov.br/ccivil_03/_ato2004-2006/2004/decreto/d5296.htm.

Brasil. (2000a). Lei $n^{o}$. 10.048, de 8 de novembro de 2000. Dá prioridade de atendimento às pessoas que especifica, e dá outras providências. http://www.planalto.gov.br/ccivil/leis/LEIS_2000/L10048.htm.

Brasil. (2000b). Lei $n^{\circ}$. 10.098, de 19 de dezembro de 2000. Estabelece normas gerais e critérios básicos para a promoção da acessibilidade das pessoas portadoras de deficiência ou com mobilidade reduzida, e dá outras providências. http://www.planalto.gov.br/ccivil/leis/LEIS_2000/L10098.htm.

Brasil (2004b). Ministério das Cidades. Brasil Acessível: implementação de políticas municipais de acessibilidade.

Calado, G. C. (2006). Acessibilidade no Ambiente Escolar: reflexões com base no estudo de duas escolas municipais de Natal-RN. 2006. 191 f. Dissertação Universidade Federal do Rio Grande do Norte - Centro de Tecnologia - Programa de Pós-graduação em arquitetura e urbanismo. Natal.

Carvalho e Castro, J. (2012). Arquitetura inclusiva: calçadas e acessibilidade. Forum da construcão. http://www.forumdaconstrucao.com.br/conteudo.php?a=35\&Cod=934.

Condorcet, B. (2006). A acessibilidade do conteúdo.2006. http://intervox.nce.ufrj.br/ bernard/VI_encontro/4_ACESSI.TXT.

Duarte, C. R. S.; Cohen, R. (2004). Afeto e Lugar: A Construção de uma Experiência Afetiva por Pessoas com Dificuldade de Locomoção. Anais do Seminário Acessibilidade no Cotidiano.

Fávero, E. A. G. (2004). Direitos das pessoas com deficiência: garantia de igualdade na diversidade. WVA.

Feijó, A. R. A. (2002). Direitos humanos e proteção jurídica da pessoa portadora de deficiência: normas constitucionais de acesso e efetivação da cidade à luz da Constituição Federal de 1988. Brasília: Ministério da Justiça, Secretaria de Estado dos Direitos Humanos.

Gil, A. C. (2008). Métodos e técnicas de pesquisa social. (6a ed). Atlas.

Gomide, A. Á., Galindo, E. P. (2013). A mobilidade urbana: uma agenda inconclusa ou o retorno daquilo que não foi. Estudos Avançados. 27 (79) $27-39$. http://dx.doi. org/10.1590/S0103-40142013000300003.

Instituto Brasileiro de Geografia e Estatística - IBGE. (2010) Censo Demográfico 2010: características gerais da população, religião e pessoas com deficiência. IBGE.

Lakatos, E., Marconi, M. de A. (1996). Técnicas de pesquisa: planejamento e execução de pesquisas, amostragens e técnicas de pesquisas, elaboração, análise e interpretação de dados. (3a ed). Atlas.

Lanchoti, J. A. (2016). Programa Municipal de Eliminação de Barreiras Arquitetônicas para o município de Ribeirão Preto.

Leite, F. P. A. (2007). O município acessível à pessoa portadora de eficiência: o direito à eliminação das barreiras arquitetônica. RCS editora.

Leite, N. B. F. (2013). Expansão urbana e seus efeitos sobre a mobilidade e acessibilidade avaliada com o auxílio dos sistemas de informação geográfica (SIG) em Teresina-PI. (Tese), Universidade Federal de Minas Gerais, Belo Horizonte.

Miotti, L. A. A. (2012). Engenharia Civil como instrumento para a acessibilidade em ambientes construídos e a realidade de calçadas e passeios urbanos. Revista Eletrônica de Engenharia Civil-REEC.

Niess, L. T. T., Niess, P. H. T. (2003). Pessoas portadoras de deficiência no direito brasileiro. Juarez de Oliveira.

Prado, A. R. A., Lopes, M. E.; Ornstein, S. W. (2010). Desenho Universal: Caminhos da Acessibilidade no Brasil. Annablume.

Quaresma, R., Romita, A. S. (2001). Comentários à legislação constitucional aplicável às pessoas portadoras de deficiência. Forense.

Santos Filho, G. M. (2010). Construindo um itinerário histórico do desenho universal: a normatização nacional e internacional da acessibilidade. São Paulo: Annablume.

Santos, M. (1988). Metamorfoses do espaço habitado. Hucitec. 
Research, Society and Development, v. 10, n. 7, e29310716526, 2021

(CC BY 4.0) | ISSN 2525-3409 | DOI: http://dx.doi.org/10.33448/rsd-v10i7.16526

Sestaro, A. C. (2008). Campanha siga essa ideia. Anais do Seminário "Os Desafios de Adequação à Lei de Acessibilidade”.

Silva, E. R. R. (2019). Acessibilidade e Mobilidade Urbana: Estudo de caso no Parque Ambiental da cidade de Codó - MA. 30 f. (Trabalho de Conclusão de Curso), Centro Universitário de Ciências e Tecnologia do Maranhão - UNIFACEMA, Caxias.

Vergara, S. C. (2005). Métodos de pesquisa em administração. Atlas. 